\title{
The Clinical Uses of Collagen-Based Matrices in the Treatment of Chronic Wounds
}

\author{
Hyee Jae Yang, Sang Yoon Kang \\ Department of Plastic Surgery, Kyung Hee University Hospital, Kyung Hee University College of Medicine, Seoul, Korea
}

\begin{abstract}
Chronic wounds are defined as wounds that fail to heal completely at the pace and order observed in normal phases of wound healing. Whereas acute wounds proceed through the normal stages of wound healing, the healing process in chronic wounds does not allow for regeneration and repair. Collagen, essential for all phases of the wound-healing cascade, only exists at lower levels in chronic wounds than in normal tissue or acute wounds, as do other extracellular matrix components. Therefore, application of collagen-based matrices is believed to modulate the chronic wound environment and contribute to wound healing in a variety of ways. The collagen-based matrices facilitate chemotaxis and migration of cells such as fibroblasts. These biomaterials provide the structural scaffold, which encourages new tissue regeneration and protects proteins involved in the process of wound healing, thereby restoring the wound environment to normal and promoting the healing. We used collagen-based matrices for the treatment of chronic wounds with various etiologies, including surgical wounds, diabetic ulcers, traumatic wounds, chronic vascular ulcers, and in preparation for skin grafts. The clinical results in the case report suggest that collagen-based matrices could be a reasonable treatment option for chronic wounds.
\end{abstract}

Keywords: Wound healing; Collagen; Extracellular matrix

\section{Introduction}

Chronic wounds are defined as wounds that fail to heal completely at the pace and order observed in normal phases of wound healing [1]. Chronic wounds continue to present a significant challenge in health care across the globe. Whereas acute wounds proceed through the normal stages of wound healing, chronic wounds often fail to achieve orderly regeneration and repair [2]. Essential in all phases of the wound-healing cascade is collagen, produced by fibroblasts and accounting for $25 \%$ of the total protein mass of mammals. Collagen also forms the structural scaffold of healing wounds. It stimulates adhesion, chemotaxis, and migration of specific cells such as macrophages and fibroblasts [3]. As there is less collagen and other extracellular matrix components in chronic wounds than in normal tissue or acute wounds, application of collagen-based matrices is believed to modulate the chronic wound environment and contribute to wound healing in a variety of ways. Although several studies have reported the use of collagen in biological dressings [4-6], its precise mechanism of action remains unclear. Here we present the clinical uses of collagen-based matrices in the treatment of chronic wounds with various etiologies. The clinical results suggest that collagen-based matrices could present a favorable treatment option for chronic wounds.

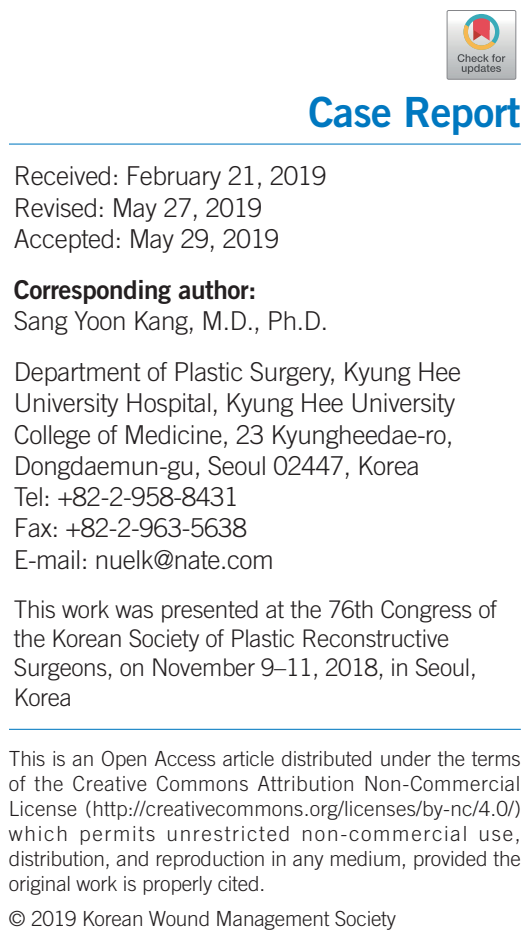

Department of Plastic Surgery, Kyung Hee University Hospital, Kyung Hee University College of Medicine, 23 Kyungheedae-ro,

Dongdaemun-gu, Seoul 02447, Korea

Tel: +82-2-958-8431

Fax: +82-2-963-5638

E-mail: nuelk@nate.com

This work was presented at the 76th Congress of the Korean Society of Plastic Reconstructive Surgeons, on November 9-11, 2018, in Seoul, Korea

This is an Open Access article distributed under the terms of the Creative Commons Attribution Non-Commercial License (http://creativecommons.org/licenses/by-nc/4.0/) which permits unrestricted non-commercial use, distribution, and reproduction in any medium, provided the original work is properly cited.

(c) 2019 Korean Wound Management Society 


\section{Cases}

The study was performed in accordance with the principles of the Declaration of Helsinki. Written informed consents were obtained.

\section{Case 1}

A 47-year-old male patient was referred to the plastic surgery department from the orthopedic surgery department. He had a $14 \mathrm{~cm} \mathrm{L-shaped} \mathrm{surgical} \mathrm{wound} \mathrm{and} \mathrm{partial} \mathrm{dehiscence} \mathrm{at} \mathrm{the}$ right knee after revision of total knee replacement for infection. Standard therapies for 1 month, including simple wound dressing with antibiotics ointment and foam dressing, had been unsuccessful (Fig. 1A). The patient had hemophilia A and hepatitis C. Previous wound cultures did not show any bacteria. Partial debridement of the wound bed was performed, followed by the application of collagen-based matrices. The collagen-based matrix was used to cover the dehiscence after debridement (Fig. 1B). The collagen-based matrix was applied every week, accompanied by debridement (Fig. 1C). During the period from the first to the third application, the wound dehiscence was filled with granulation tissue, at which point we changed to dressing with potadine-soaked gauze once daily (Fig. 1D). Three weeks after the third application, the wound was nearly healed. At 4 months follow-up, the wound was completely healed (Fig. 1E).

\section{Case 2}

A 54-year-old male patient was referred from the general surgery department for wound management. He had a 3-monthold wound at the right lateral malleolar area. The wound was a 2-cm round-shaped diabetic ulcer half-covered with yellowish granulation tissue (Fig. 2A). The patient had hypertension and diabetes mellitus. Previous wound cultures did not show any bacteria. The first application of collagen-based matrix was performed after debridement (Fig. 2B) and the second application was 2 weeks later. Four weeks after the second application, about $70 \%$ of the wound was epithelized (Fig. 2C). The third application was performed on the unhealed portion of the wound. The wound was completely healed at 2 months follow-up (Fig. 2D).

\section{Case 3}

A 71-year-old male patient was referred from the general sur-
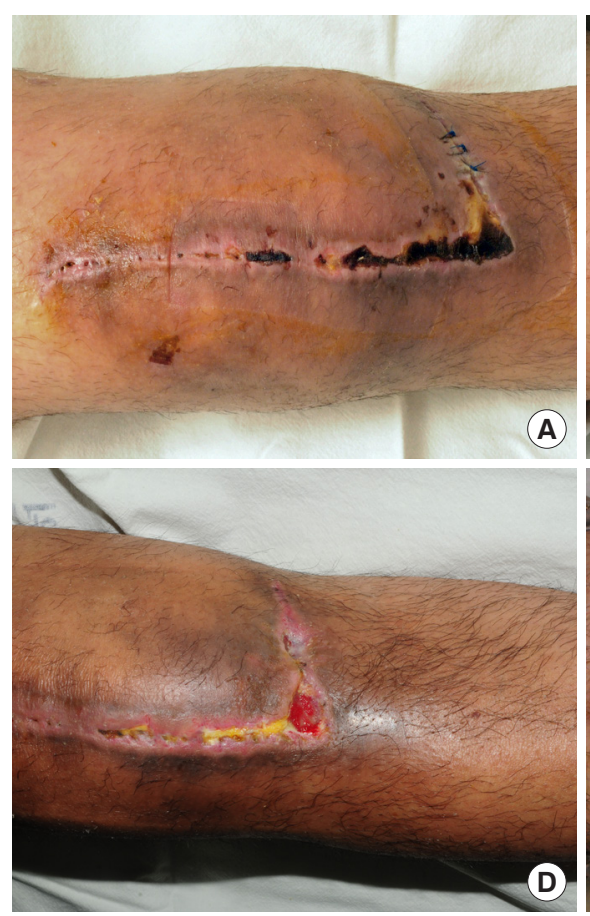
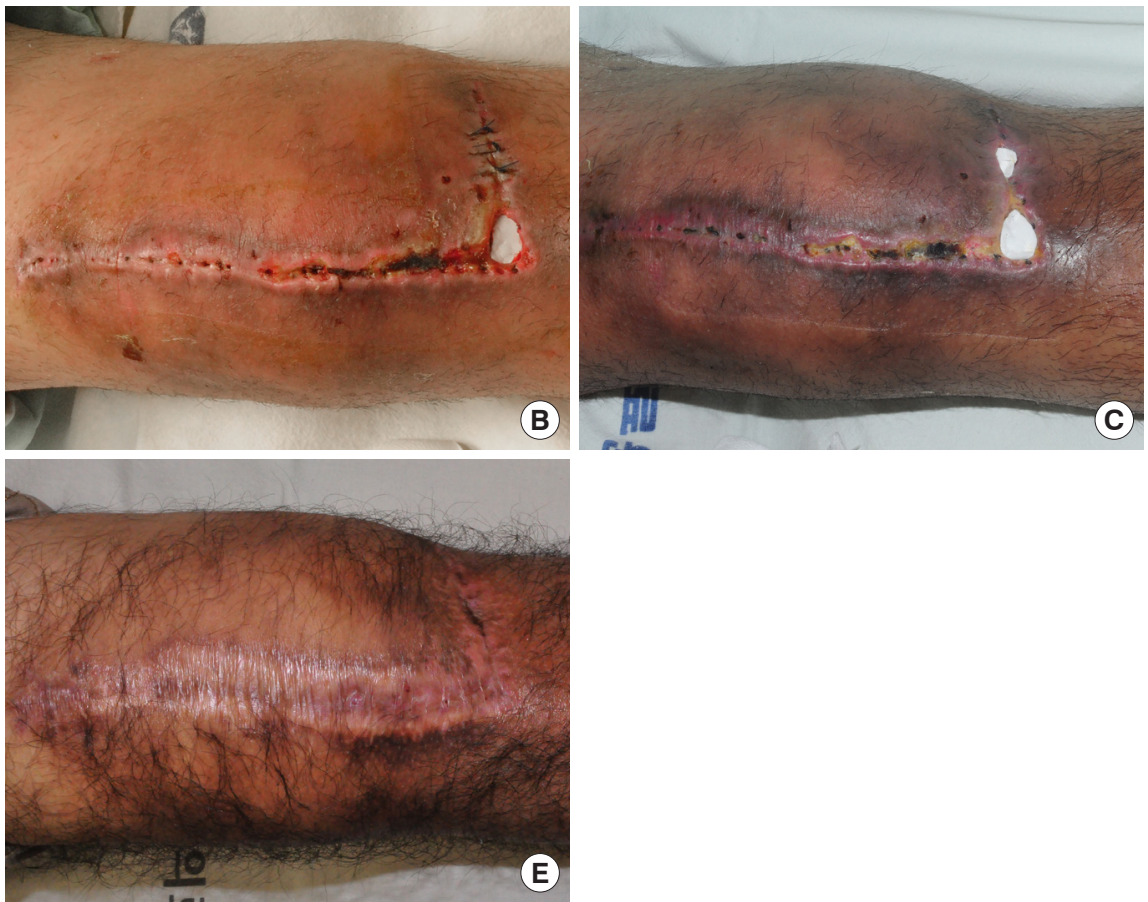

Fig. 1. A 47-year-old male with a surgical wound. (A) A 14-cm L-shaped surgical wound and partial wound dehiscence at the right knee. (B) The first application of collagen-based matrix at the dehiscence. (C) The second application 1 week after the first application. (D) One week after the third application, the wound dehiscence filled with granulation tissue. (E) At the 4-month follow-up, the wound was completely healed. 

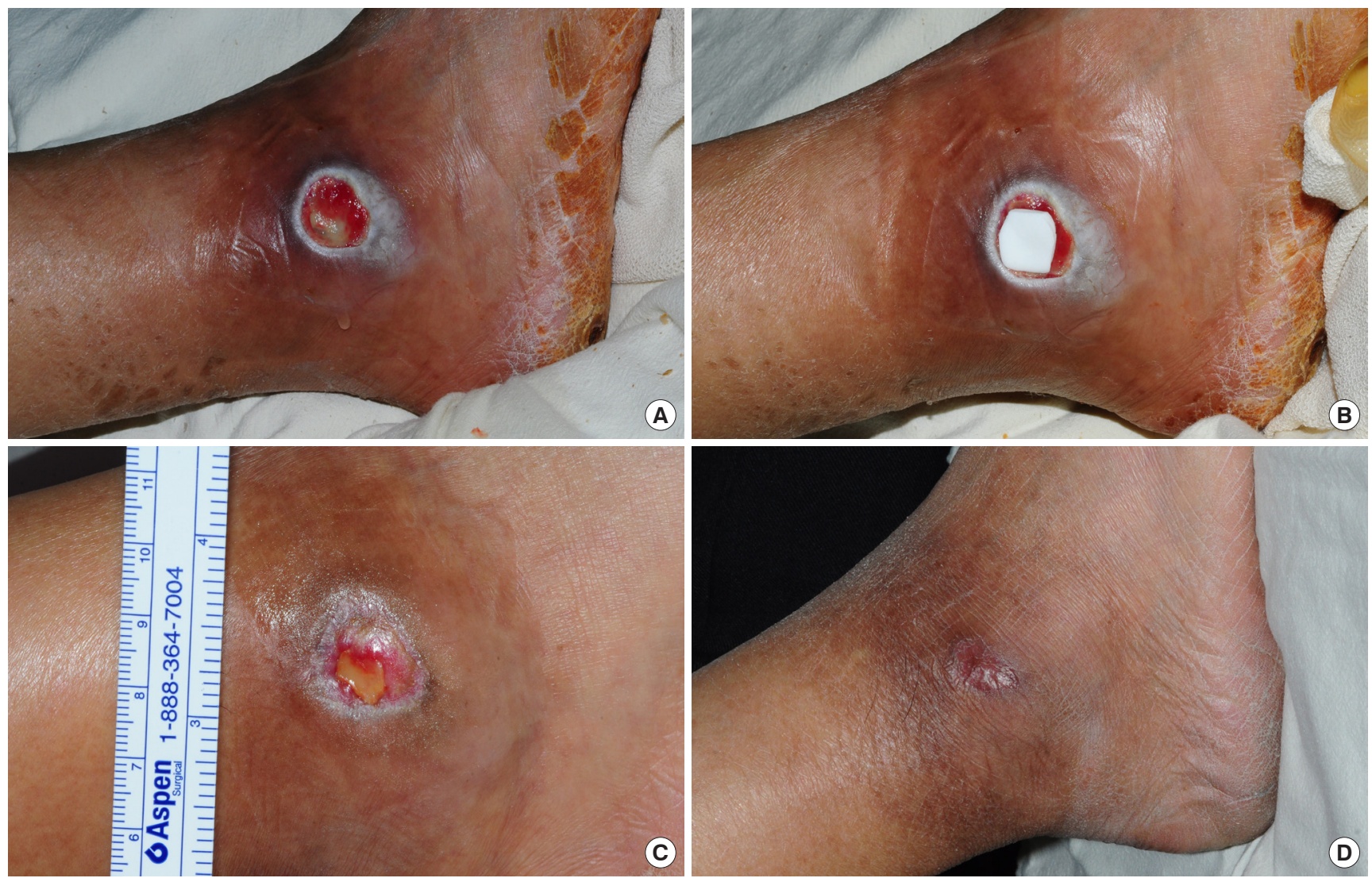

Fig. 2. A 54-year-old male with a diabetic ulcer. (A) A 2-cm round-shaped ulcer at the right lateral malleolar area. (B) Application of collagen-based matrix after debridement. (C) Four weeks after the second application, about $70 \%$ of the wound was epithelized. (D) At the 2-month follow-up, the wound was completely healed.
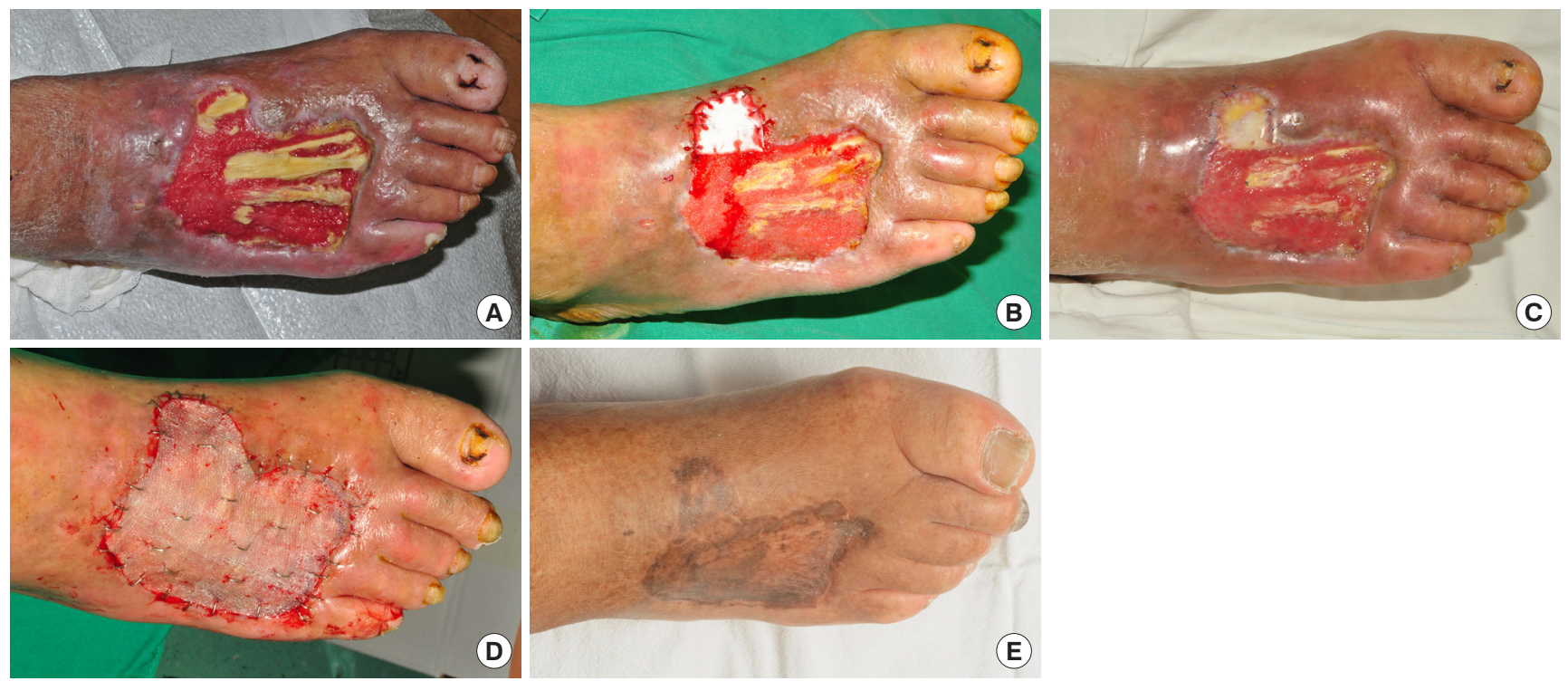

Fig. 3. A 71-year-old male with a skin defect. (A) An $8 \times 7 \mathrm{~cm}$ skin defect with exposure of the tendons at the dorsum of the right foot. (B) Application of collagen-based matrix on the extensor hallucis longus tendon. (C) The clinical photograph before split-thickness skin graft (STSG) coverage 2 weeks after the application of collagen-based matrix. (D) STSG coverage was completed. (E) Completely healed wound at long-term follow-up. 

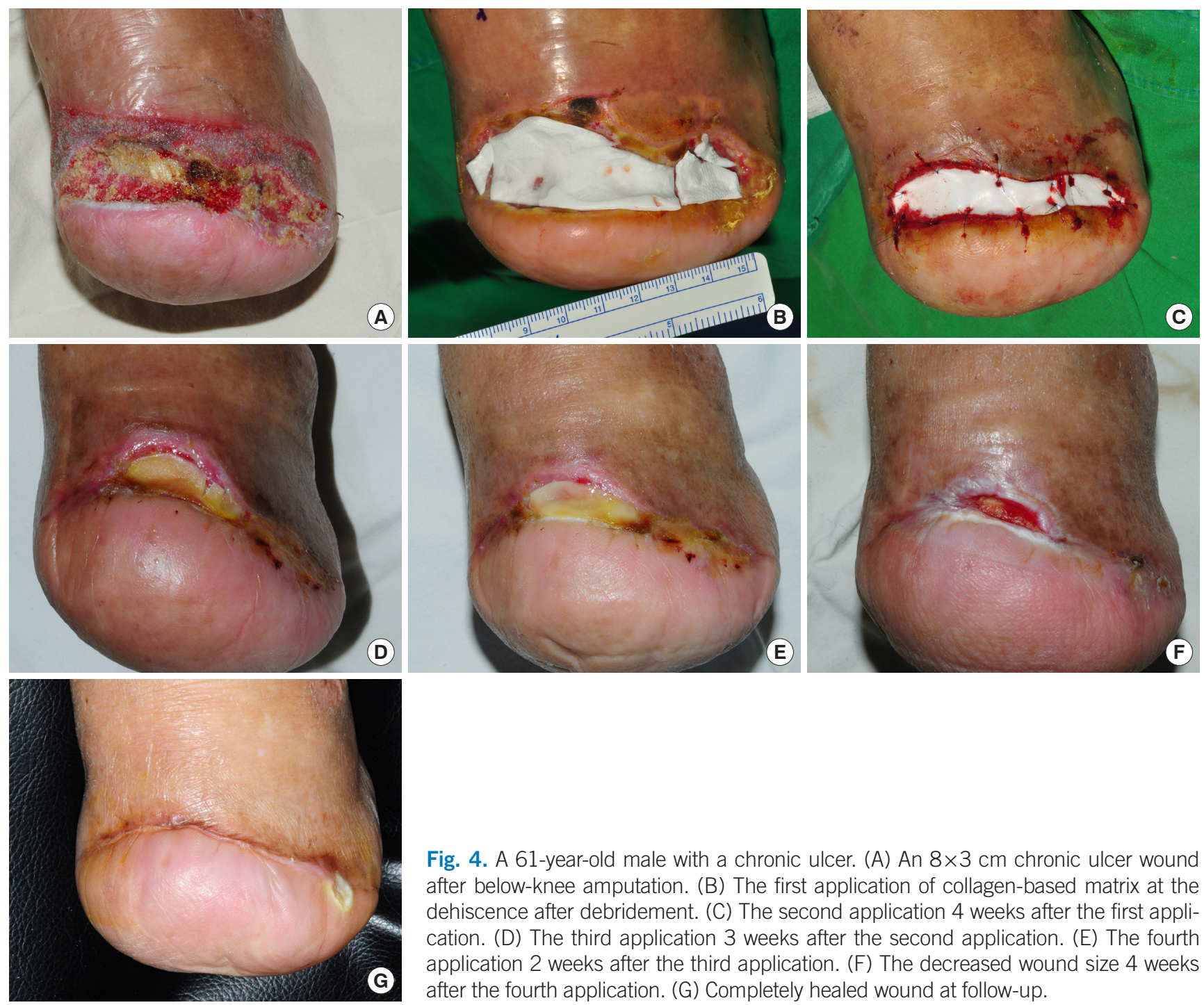

Fig. 4. A 61-year-old male with a chronic ulcer. (A) An $8 \times 3 \mathrm{~cm}$ chronic ulcer wound after below-knee amputation. (B) The first application of collagen-based matrix at the dehiscence after debridement. (C) The second application 4 weeks after the first application. (D) The third application 3 weeks after the second application. (E) The fourth application 2 weeks after the third application. (F) The decreased wound size 4 weeks after the fourth application. (G) Completely healed wound at follow-up.

gery department for management of an $8 \times 7 \mathrm{~cm}$ skin defect with exposed tendons at the dorsum of his right foot. The wound had persisted for 2 months. The wound developed after a bypass surgery for atherosclerosis obliterans (ASO) of the right anterior tibial artery (Fig. 3A). In addition to ASO, the patient also had hypertension and diabetes mellitus. Previous wound cultures did not show any bacteria. Prior to undergoing split-thickness skin graft (STSG) coverage, the wound bed was prepared to ensure survival of the skin grafted on tissues with limited blood supply, such as tendons. The collagenbased matrix was applied to the extensor hallucis longus tendon (Fig. 3B). STSG coverage was undertaken 2 weeks after the application of collagen-based matrix (Fig. 3C and D). At 2 months follow-up, the wound was completely healed. The surgical area was well maintained after 2 years (Fig. 3E).

\section{Case 4}

A 61-year-old male patient was referred from the general surgery department for management of a chronic ulcer wound after below-knee amputation due to diabetes mellitus foot (Fig. 4A). The wound had persisted for 3 months. The patient had hypertension and diabetes mellitus. Previous wound cultures did not show any bacteria. The first application of collagenbased matrix was performed after debridement (Fig. 4B) and the second application was 4 weeks later (Fig. 4C). The third and fourth applications were 3 weeks and then 2 weeks later, respectively (Fig. 4D and E). Four weeks after the fourth application, the size of the wound decreased and healthy granulation tissue was observed at the wound bed (Fig. 4F). The wound was completely healed at 6 months follow-up (Fig. 4G). 


\section{Discussion}

Collagen accounts for $25 \%$ of the total protein mass of mammals. Collagen is produced by fibroblasts, which are predominant in the proliferative phase of normal wound healing process. Collagen acts as the structural scaffold of the healing wound and stimulates adhesion, chemotaxis, and migration. The collagen fiber bundles stabilized by covalent cross-links produce mechanical tension vectors and provide the tensile strength to the skin [7].

However, the recruitment of fibroblasts and the expression of collagen are suppressed in chronic wounds [8,9]. Matrix metalloproteinase (MMP) and elastase also affect the collagen levels in chronic wounds. MMPs proteolytically degrade intact collagen and partially degrade collagen fragments. Elastase, a nonspecific protease, converts pro-MMPs to active MMPs and degrades collagen by binding to it. In acute wound healing, the dynamic synthesis and degradation of collagen by MMPs proceed normally. However, in chronic wounds, MMP levels are elevated and MMP inhibitors are decreased [10,11]. Also, the high elastase activity in chronic wounds contributes to the elevation of MMPs. All of these result in decreased collagen deposition and increased collagen degradation.

Many dressing materials have been developed to protect the healing wound from infection and to promote the wound healing process. Among them, collagen products have been used on recalcitrant wounds and chronic ulcers. The collagen products may be derived from multiple sources, including bovine and porcine collagen, and are not intended to fully replace new production of collagen in wounded tissue. Nevertheless, the products are believed to help attract cell types critical to wound healing while reducing negative effectors such as free radicals and proteases [12].

Among the many kinds of collagen-based dressings, we used ProHeal (MedSkin Solutions Dr. Suwelack AG) which is a sterile, biocompatible wound dressing comprised of natively structured bovine collagen fibers. The fibrous structure of the ProHeal matrix allows for good stability against enzymatic degradation. In addition, ProHeal enhances epithelization and stimulates collagen expression [13]. The collagen-based matrices are used on a variety of wounds, including surgical wounds, diabetic ulcers, traumatic wounds, chronic vascular ulcers, pressure ulcers, and in preparation for skin grafts.

In general, the wound bed is debrided prior to the application of collagen-based matrices. In the cases presented here, these materials, along with antibiotic ointment, were applied directly upon the wound bed and were covered with secondary dressing. The levels of residual collagen materials in the wound determined the rate of application. If there were collagen residues in the wound, only antibiotic ointment was applied, followed by the secondary dressing. Collagen-based dressing was applied when no residual collagen materials were detected in the wound. The rate of application was on average once every week. The application of collagen materials was discontinued after formation of healthy granulation tissue and epithelization in the wound. Formation of healthy granulation tissue means that the wound is healing normally and has entered the proliferation phase. If the appropriate environment is maintained, the remodeling phase of the wound healing begins.

The collagen-based matrices facilitate chemotaxis and migration of cells such as fibroblasts. These biomaterials provide the structural scaffold which encourages new tissue regeneration and protects proteins involved in the wound healing process [14].

In conclusion, we used collagen-based matrices for the treatment of chronic wounds with various etiologies, including surgical wounds, diabetic ulcers, traumatic wounds, chronic vascular ulcers, and in preparation for skin grafts. Wounds were successfully treated with the collagen-based matrices. The clinical results in the case report suggest that collagen-based matrices could be a reasonable treatment option for chronic wounds.

\section{Conflict of interest}

No potential conflicts of interest relevant to this article are reported.

\section{Acknowledgments}

Hyee Jae Yang https://orcid.org/0000-0001-8068-8770

Sang Yoon Kang https://orcid.org/0000-0002-6299-6371

\section{References}

1. Frykberg RG, Banks J. Challenges in the treatment of chronic wounds. Adv Wound Care (New Rochelle) 2015;4:560-82.

2. Serena TE. Use of epidermal grafts in wounds: a review of an automated epidermal harvesting system. J Wound Care 2015; 24(4 Suppl):30-4.

3. Tracy LE, Minasian RA, Caterson EJ. Extracellular matrix and dermal fibroblast function in the healing wound. Adv Wound 
Care (New Rochelle) 2016;5:119-36.

4. Mulder G, Lee DK. A retrospective clinical review of extracellular matrices for tissue reconstruction: equine pericardium as a biological covering to assist with wound closure. Wounds 2009;21:254-61.

5. Eisenbud D, Huang NF, Luke S, et al. Skin substitutes and wound healing: current status and challenges. Wounds 2004; 16:2-17.

6. Fleck CA, Chakravarthy D. Understanding the mechanisms of collagen dressings. Adv Skin Wound Care 2007;20:256-9.

7. Fleck CA, Simman R. Modern collagen wound dressings: function and purpose. J Am Col Certif Wound Spec 2011;2: 50-4.

8. Falanga V. Chronic wounds: pathophysiologic and experimental considerations. J Invest Dermatol 1993;100:721-5.

9. Hansen SL, Young DM, Boudreau NJ. HoxD3 expression and collagen synthesis in diabetic fibroblasts. Wound Repair Regen 2003;11:474-80.
10. Wysocki AB, Staiano-Coico L, Grinnell F. Wound fluid from chronic leg ulcers contains elevated levels of metalloproteinases MMP-2 and MMP-9. J Invest Dermatol 1993;101:64-8.

11. Ladwig GP, Robson MC, Liu R, et al. Ratios of activated matrix metalloproteinase-9 to tissue inhibitor of matrix metalloproteinase-1 in wound fluids are inversely correlated with healing of pressure ulcers. Wound Repair Regen 2002;10: 26-37.

12. Han G, Ceilley R. Chronic wound healing: a review of current management and treatments. Adv Ther 2017;34:599-610.

13. Haycocks S, Chadwick P, Cutting KF. Collagen matrix wound dressings and the treatment of DFUs. J Wound Care 2013; 22:369-70.

14. Cullen B, Smith R, McCulloch E, et al. Mechanism of action of PROMOGRAN, a protease modulating matrix, for the treatment of diabetic foot ulcers. Wound Repair Regen 2002;10: 16-25. 